\title{
Mecatrónica educativa: soporte de la enseñanza-aprendizaje de educación básica en Hidalgo
}

\section{Educational mechatronics: support for teaching-learning of basic education in Hidalgo}

\author{
J. C. González-Islas (D) G. Godínez-Garrido (iD*, A. González-Rosas (D), B.A. Ortega-Marín (iD \\ Área Electromecánica Industrial, Universidad Tecnológica de Tulancingo, 43645, Tulancingo, Hidalgo, México.
}

\begin{abstract}
Resumen
México se encuentra inserto en el avance científico, tecnológico y de innovación para el desarrollo de proyectos que resuelven necesidades globales del sector educativo. Hidalgo no es la excepción, por lo que docentes y estudiantes de la Universidad Tecnológica de Tulancingo se han sumado a este esfuerzo con el propósito de fomentar el interés científico, tecnológico y de innovación de estudiantes de educación básica de la entidad y fortalecer el proceso de enseñanza-aprendizaje de dicho nivel educativo. En este artículo se presenta la metodología de implementación y el método de desarrollo de prototipos y material didáctico de un enfoque novedoso basado en mecatrónica educativa para la educación básica secundaria de la entidad. Finalmente, se describen de manera cualitativa los resultados más significativos de la puesta en marcha de este enfoque de 2016 a la fecha en diversos escenarios, los cuales permiten determinar la factibilidad pedagógica y viabilidad tecnológica para su implementación a mayor escala en el estado.
\end{abstract}

Palabras Clave:

Mecatrónica educativa, educación básica secundaria, proceso enseñanza-aprendizaje, tecnología e innovación educativa

\begin{abstract}
At present, Mexico finds itself in the middle of a great advancement in science, technology, and innovation via the development of projects which fulfil current needs and are supported by the educational sector. The state of Hidalgo is no exception and as a result, educators and students alike from the Universidad Tecnológica de Tulancingo have joined the effort with a goal of encouraging interest by basic education students in the areas of science, technology, and innovation and by strengthening the teaching and learning processes at the basic education level. In this article, we will present the methodology of implementation as well as development methods for prototypes and didactic materials with a new focus based on educational mechatronics designed for a basic education level at each institution. Finally, we will outline the most significant qualitative results since the 2016 launch of this approach in diverse scenarios, an analysis which will permit us to determine the pedagogical and technological feasibility of such an approach on a statewide scale.
\end{abstract}

Keywords:

Educational mechatronics, basic education, teaching and learning processes, educational technology and innovation

\section{Introducción}

La globalización y la complejidad que hoy el mundo representa para el ser humano, implica que todas las personas tengan competencias (habilidades, conocimientos y valores) para resolver problemas de alta complejidad, por eso es muy importante en la sociedad formar al estudiante como un ciudadano integral que identifique los retos a su alrededor, diferencie y potencie entre sus herramientas lo que sabe, y lo que hace con lo que sabe con base en su criterio y decisión (Luque-Vega et al., 2019), tal y como se plantea indirectamente en este proyecto.

Finlandia, uno de los líderes mundiales en términos de educación (Lonka, 2020), desde inicios del nuevo milenio, ha impulsado la educación tecnológica e incluyente. Uno de los enfoques empleados ha sido la modernización de la educación

*Autor para la correspondencia: gildardo.godinez@utectulancingo.edu.mx

Correo electrónico: juancgonzalez@utectulancingo.edu.mx (Juan Carlos González-Islas), gildardo.godinez@utectulancingo.edu.mx (Gildardo Godínez-Garrido), agonzalez@utectulancingo.edu.mx_(Angelina González-Rosas), blancaortega@utectulancingo.edu.mx (Blanca Andrea Ortega-Marín). 
tecnológica en las instituciones educativas apoyándose en parte en la destreza manual. Este sistema ha incorporado los avances tecnológicos y la innovación en beneficio de la sociedad desde la formación de sus estudiantes, lo que les ha traído tan buenos resultados (Halinen \& Järvinen, 2008, Kananoja, 2009).

La mayoría de los países más desarrollados y exitosos del planeta tienen una estructura educativa consolidada y sin embargo, desde hace tiempo han tomado acciones para innovar sus modelos educativos, tal es el caso de Estados Unidos de América a través del departamento de educación con el programa para la educación basado en Ciencia, Tecnología, Ingeniería y Matemáticas (STEM por sus siglas en inglés: Science Technology Engineering and Mathematics), el cual es una propuesta integral de enseñanza aprendizaje de estas cuatro áreas, para preparar a sus estudiantes como futuros ciudadanos exitosos del mundo (King et al., 2016, Krach, 2017, Cibrián-Anaya et al., 2019). En el mismo sentido se encuentra el proyecto realizado en España, Finlandia, Colombia, Uruguay, Portugal, Italia, Reino Unido y Australia, junto con dos instituciones científicas, que durante tres años efectuaron proyectos interactivos apoyados con tecnología digital (Scolari, 2018).

Otro de los países con grandes avances tecnológicos es Japón, ellos ya han iniciado el camino hacia la innovación tecnológica en la educación desde hace más de una década. Un proyecto exitoso que han emprendido en este país son las Preparatorias Super Científicas (SSHS por sus siglas en inglés: Super Science High Schools), donde se forman los ciudadanos que dirigen los avances científicos y tecnológicos de esta nación (Kawamoto, 2015).

Hoy en día, la tendencia mundial en el ámbito educacional es la Educación 4.0, la cual se debe entender desde el ámbito de la Revolución Industrial 4.0 (IR por sus en inglés: Industrial Revolution) en la que el avance de las nuevas tecnologías disruptivas reduce los enlaces entre los entornos físico, digital y biológico. La IR 4.0 no solo impacta en los negocios, el gobierno y las personas, sino también lo hace en la educación (Educación 4.0). Las tendencias relacionadas con la Educación 4.0 y que conciernen con la propuesta presentada en este trabajo de investigación, son el aprendizaje interactivo y centrado en el estudiante basado en tecnologías emergentes como el Internet de las cosas (IoT), las TICS o la inteligencia artificial (Hussin, 2018).

Para el caso de México la propuesta de la reforma educativa ha sido un gran paso hacia una educación de calidad, para promover el desarrollo, capacitación tecnológica y evaluación de docentes y directivos (Secretaría de Educación Pública (SEP), 2017). Para ello ha integrado como pilar una propuesta sólida innovadora basada en la educación tecnológica, tal y como la que se presenta en este proyecto. La cual lleva a aprender a aprender; mediante la aplicación del conocimiento para la resolución de problemas de la vida diaria.

Otro de los mecanismos que permitirá a nuestro país tener una sociedad próspera e incluyente es hacer del desarrollo científico, tecnológico y la innovación pilares para el progreso económico y social, articulando los esfuerzos que se realizan en los sectores público, privado y social, para la resolución de los problemas complejos que presenta en la actualidad el mundo global; tal y como se lo han planteado el gobierno federal y estatal a través del Consejo Nacional de Ciencia y Tecnología (CONACyT) y del Consejo de Ciencia Tecnología e Innovación de Hidalgo (CITNOVA), respectivamente. Como por ejemplo el modelo interactivo basado en las Tecnologías de Información y Comunicación (TICs), el cual favorece la implementación de un modelo educativo basado en la construcción del conocimiento por los estudiantes (López-García et al., 2019).

\subsection{La educación en el estado de Hidalgo}

En Hidalgo, la educación se ha centrado en fortalecer la articulación con la Educación Básica y Media Superior, con el objetivo de desarrollar las competencias que demandan los sectores industrial y social hoy día, ampliando el acceso a la tecnología educativa través de la robótica educativa (Gobierno del Estado de Hidalgo, 2016, Cibrián-Anaya et al., 2019). Además, es clara la necesidad de generar estrategias didácticas y pedagógicas que coadyuven a disminuir la deserción y fomentar el interés por la ciencia, tecnología e innovación desde la educación básica en la entidad.

El antecedente de este trabajo de investigación data de 2012 con la instalación de la Academia Estatal de Robótica de Hidalgo, auspiciada por el gobierno Estatal y orientada a promover la generación y transferencia de conocimientos de los profesores, impulsando a los estudiantes a apoyar en los quehaceres cotidianos de los sectores industrial y social de la entidad mediante la robótica educativa.

Desde 2013, los estudiantes de Ingeniería en Mecatrónica de esta institución han realizado varios proyectos orientados a la tecnología y robótica educativa, con el objetivo de consolidar los conocimientos de su perfil en aplicaciones prácticas; para la solución de problemas de los ámbitos social e industrial de la región. Dichas propuestas han participado en múltiples concursos de prototipos como Leamos la Ciencia, Creatividad e Ingenio (Museo El Rehilete), Concurso Nacional de Innovación y Emprendimiento, entre otros, destacando por su grado de innovación y pertinencia. Varios de esos desarrollos son la base conceptual de los prototipos del enfoque de mecatrónica educativa propuestos en este trabajo.

El objetivo principal de esta investigación en su primera etapaes fomentar el interés científico, tecnológico y de innovación para que los estudiantes de educación básica secundaria comprendan los problemas reales; mediante el desarrollo e integración de prototipos interactivos mecatrónicos y de material didáctico, apoyando el proceso de enseñanza- aprendizaje.

Derivado de lo anterior, este trabajo de investigación se centra en la implementación de un enfoque basado en mecatrónica educativa para la educación básica del estado de Hidalgo. Para describir este trabajo, el artículo está organizado de la siguiente forma: 1. Introducción, trabajo relacionado, detección de necesidades y objetivo. 2. Materiales y métodos para la implantación del enfoque. 3. Prototipos mecatrónicos interactivos. Apartado 4. Resultados y discusión y 5. Conclusiones y trabajo futuro, respectivamente.

\section{Materiales y métodos}

En esta sección se describe de manera general la metodología de cómo fue desarrollado e implementado en diversos escenarios el enfoque didáctico basado en mecatrónica educativa.

\subsection{Planteamiento del enfoque a la escuela secundaria para su implementación}

Derivado de los antecedentes, la experiencia de los colaboradores del equipo de trabajo y detección del área de oportunidad de impacto del área técnica, el punto de inicio de este proyecto se dio con el planteamiento de la implementación del enfoque basado en mecatrónica educativa mediante un taller en una escuela de nivel secundaria en el ciclo escolar 2016-2017, ubicada en Tulancingo de Bravo, estado de Hidalgo. 


\subsection{Planeación del taller}

Una vez autorizada la implantación del enfoque, se realizó la planeación del taller basado en mecatrónica educativa y centrado en los contenidos curriculares de la secundaria, previo a ofertarlo a los estudiantes en el ciclo escolar.

\subsection{Implementación del enfoque y desarrollo de prototipos y material didáctico}

Durante el ciclo escolar 2016-2017, mediante la impartición del taller a 124 alumnos de los 3 grados de ese nivel educativo, de 30 horas a la semana se implementó el enfoque propuesto. Los prototipos mostrados en la sección 3. Prototipos mecatrónicos interactivos, forman parte del catálogo del modelo, fueron desarrollados y validados durante el ciclo escolar con la colaboración significativa de los estudiantes. Asimismo, se generó el material didáctico de propósito específico para complementar el taller. De igual forma, continua y periódicamente se evaluaron las competencias de los estudiantes; con base en una rúbrica y la calificación se registró en la materia de taller.

\subsection{Análisis de resultados}

Derivado de la naturaleza del proyecto, se requiere un análisis formal para la evaluación y análisis de resultados. Si bien la encuesta satisfacción del cliente es una métrica de rendimiento empresarial de uso común, es posible evaluar a los alumnos como un cliente (Dawes et al., 2020). Sin embargo, en este trabajo de investigación, solo se realizó un análisis cualitativo basado en la percepción de los estudiantes y profesores, considerando las siguientes preguntas y su desempeño durante el desarrollo del taller.

- ¿Cuáles son los temas que se emplean en el uso de los prototipos mecatrónicos?

- ¿Cuál es la diferencia entre la enseñanza convencional y la mecatrónica educativa?

- ¿Qué tan útil es la mecatrónica educativa para resolver problemas de la vida real?

- ¿Cuál es tu percepción del uso de la mecatrónica educativa para fortalecer el proceso de enseñanza-aprendizaje en la educación básica?

Además, se disciernen resultados significativos, los cuales se discuten a detalle en la sección de resultados y discusión.

\subsection{Determinación de la madurez tecnológica con base en la metodología TRL.}

La metodología TRL (por sus siglas en inglés: Technology Readiness Level) de la NASA, es un método para estimar la madurez tecnológica de un programa o producto, que examina conceptos, requerimientos tecnológicos y capacidades de demostración (Olechowski et al., 2020). Las etapas de maduración tecnológica, según esta metodología son:

- Nivel 0. Investigación científica básica,

- Nivel 1. Investigación aplicada-tecnológica básica,

- Nivel 2. Validación conceptual,

- Nivel 3. Prueba de concepto II,

- Nivel 4. Desarrollo tecnológico,

- Nivel 5. Componentes integrados que dan lugar a que la configuración del sistema sea similar a su aplicación final,
- Nivel 6. Demostración tecnológica: Sistema de ingeniería en validación en ambiente en condiciones relevantes a las reales operativas. Aún a nivel prototipo,

- Nivel 7. Comisionamiento de sistemas. Prototipo completo demostrado en ambiente relevante,

- Nivel 8. Sistema final completo y evaluado a través de pruebas y demostraciones y

- Nivel 9. Operación del sistema.

En este trabajo se ha usado la metodología TRL, que determina el grado de madurez tecnológica de un prototipo, analizándolo y validándolo para determinar su nivel de avance de acuerdo con los niveles descritos anteriormente.

\subsection{Implementación del enfoque en otros escenarios}

Derivado de la factibilidad pedagógica y la viabilidad tecnológica del enfoque basado en prototipos mecatrónicos interactivos, como herramienta didáctica en el proceso enseñanzaaprendizaje de educación básica secundaría, en 2017 se realizó una propuesta a la Secretaría de Educación Pública de Hidalgo (SEPH) para implementar el enfoque de manera similar al de la prueba piloto en escuelas secundarias específicas del estado. En 2018, en conjunto con la Universidad Politécnica Metropolitana de Hidalgo, se inició la implantación del enfoque, orientado a robótica aérea educativa con la impartición del curso: Fortalecimiento de la enseñanza de las matemáticas, ciencias naturales y tecnologías, a 33 profesores de educación básica secundaria de todas las regiones del estado de Hidalgo. En 2019, se dio continuidad al proyecto.

En el mismo sentido, en la Universidad Tecnológica de Tulancingo, estos prototipos han sido empleados para guiar y motivar a los alumnos que asisten al taller de robótica de la institución en su replicación y mejora. Además, esta iniciativa ha impulsado en la institución el desarrollo de prototipos didácticos para el proceso de enseñanza-aprendizaje de educación superior, particularmente en el Programa Educativo (PE) de Terapia Física, mediante la creación de ambientes virtuales para conceptualizar contenidos disciplinares.

\section{Prototipos mecatrónicos interactivos}

En esta sección se describe la metodología general del desarrollo y validación de algunos de los prototipos empleados en el enfoque basado en mecatrónica educativa para la educación básica a nivel secundaria. Sin embargo, dicha metodología puede ser usada para el desarrollo de estos mismos sistemas con otro propósito.

1. Diseño conceptual y análisis funcional del prototipo,

2. Programación del firmware en los prototipos,

3. Simulación de los sistemas de control y visualización, para validar el funcionamiento propuesto en el diseño conceptual,

4. Armado de prototipado del sistema de control y visualización,

5. Fabricación e instrumentación de la tarjeta impresa de circuito $(\mathrm{PCB}) \mathrm{y}$

6. Puesta en marcha, detección y corrección de errores, para la validación de hardware del prototipo con base en el diseño conceptual. 
Una descripción funcional de algunos de los prototipos en este trabajo de desarrollo tecnológico, se encuentra a continuación. Cabe mencionar que fueron realizados con herramientas de código abierto y versiones estudiantiles de software, así como librerías de uso educativo. En todos los casos se diseñaron y realizaron las tarjetas de control, empleando microcontroladores de arquitectura abierta y software de acceso libre. Debido a que los prototipos incorporan herramientas disponibles para aplicaciones móviles y que pueden incorporarse tecnologías de IoT, TICs y cómputo en la nube, es posible conceptualizar estos sistemas dentro del paradigma de la IR 4.0 y por ende de la educación 4.0.

El primer prototipo mecatrónico propuesto en este trabajo, ver Figura 1, es un brazo robótico manipulador interactivo de tres grados de libertad (GDL); controlado por dos joysticks y que puede manejarse alternativamente con una aplicación en un dispositivo móvil.

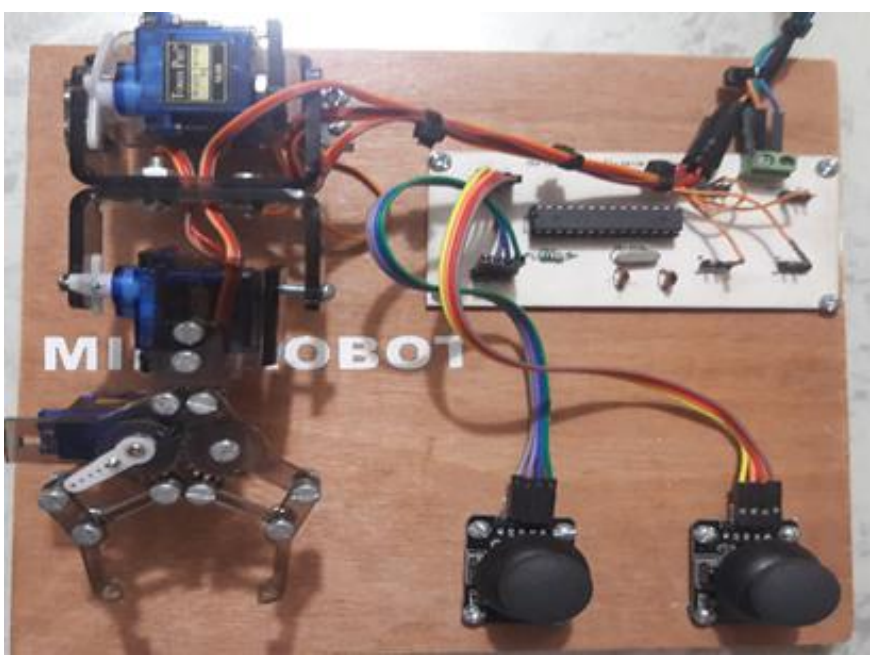

Figura 1: Brazo Robótico manipulador de 3 GDL (ElizaldeHuerta, 2017).

Con este sistema el alumno relaciona de manera didáctica e interactiva los conocimientos y leyes fundamentales de las áreas de la física, como la mecánica, estática, dinámica, electricidad, magnetismo; y matemáticas, aritmética y geometría. Esta aplicación tecnológica-práctica se adapta a un sistema robótico real, que puede ser empleado en el sector productivo en muchos de los procesos de manufactura actuales. El siguiente sistema -en la Figura 2- presenta un robot móvil seguidor de líneas blancas con fondo oscuro.

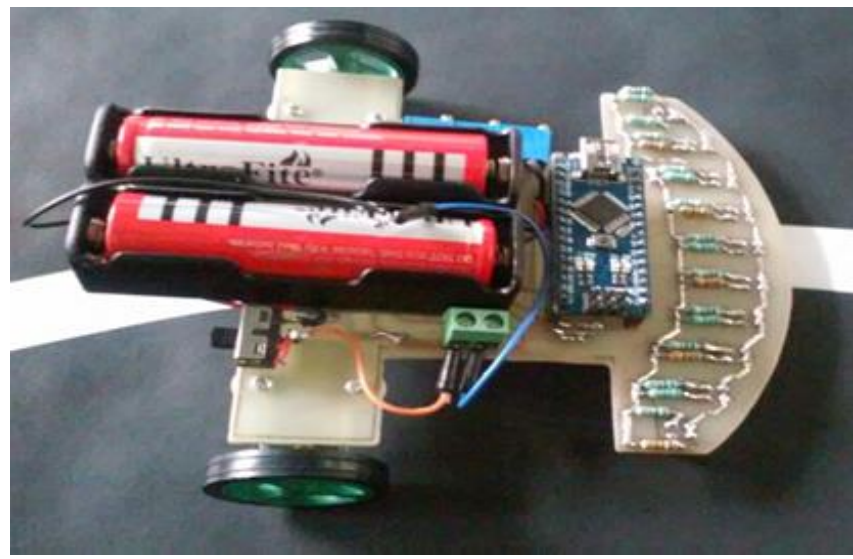

Figura 2: Robot móvil seguidor de líneas.
Mediante un sistema robótico móvil de este tipo el estudiante puede aprender y/o consolidar sus conocimientos referentes a señales, electricidad, magnetismo y mecánica, y determinar su aplicación práctica en la vida real, como lo sería un almacén inteligente, por ejemplo. En la Figura 3 se presenta un sistema de monitoreo de temperatura, con una pantalla LCD en la que se despliega la temperatura en ${ }^{\circ} \mathrm{C}$ y ${ }^{\circ} \mathrm{F}$. Una de las cuatro variables industriales más monitoreadas y controladas en los sectores productivo y social es la temperatura, por lo que es de suma importancia que un alumno desde el nivel básico determine su utilidad y principios de funcionamiento de la variable en los dispositivos, de aplicación en el sector alimenticio, como ejemplo.

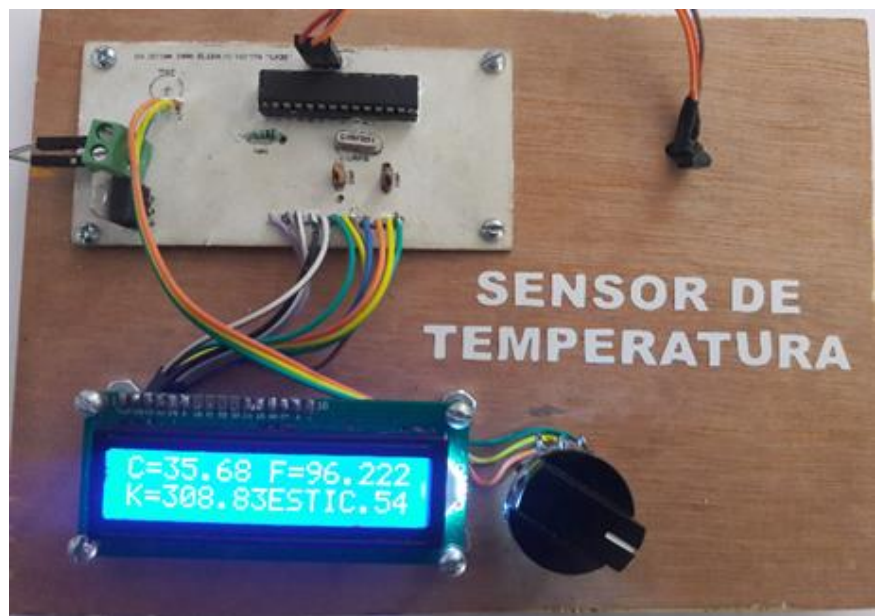

Figura 3: Sistema de monitoreo de temperatura. (ElizaldeHuerta, 2017).

Los temas relacionados con la física y matemáticas, particularmente con relación a variables físicas, metrología y álgebra pueden ser fortalecidas con sistemas como estos. Si bien la humedad no es una variable física básica, hoy en día, en algunas partes del mundo y sobre todo en los sistemas productivos utilizados en los invernaderos, el monitoreo y control de la humedad es de suma importancia. La Figura 4, muestra la aplicación real de un sistema de monitoreo de humedad con una pantalla de cristal.

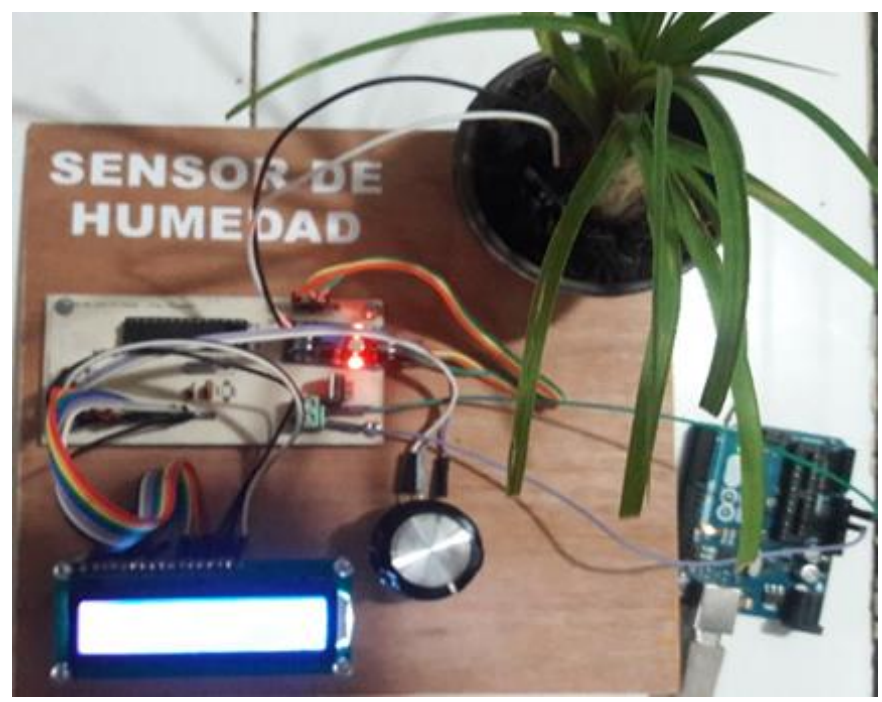

Figura 4: Sistema de monitoreo de humedad (ElizaldeHuerta, 2017). 
Por otra parte, el sonido y el ultrasonido son dos de los fenómenos físicos de mayor interés en la actualidad por lo que se hacen necesarios prototipos como los mostrados en las Figuras 5 y 6, respectivamente. La primera, se centra en detectar el sonido y mediante una señal luminosa; indicar la presencia de este. En el segundo se detecta la proximidad de un objeto, basado en el principio de funcionamiento ultrasónico. El primero es aplicable en los sistemas de seguridad y el segundo en los sistemas inteligentes de estacionamiento, por ejemplo.

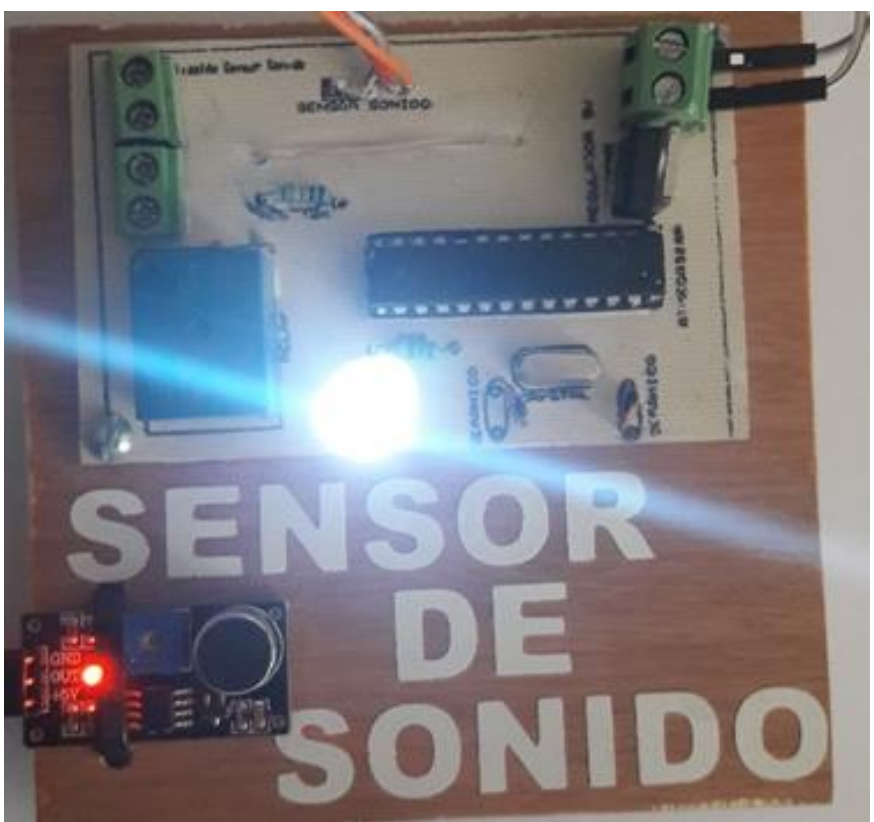

Figura 5: Sistema de monitoreo de sonido (Elizalde-Huerta, 2017).

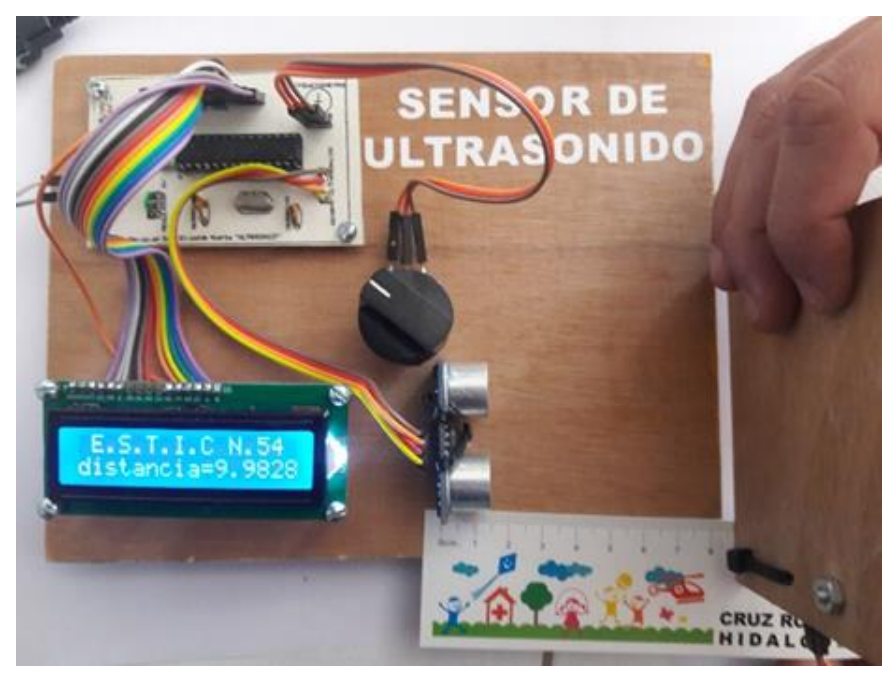

Figura 6: Sistema ultrasónico para sensar la proximidad (Elizalde-Huerta, 2017).

Otros de los temas es que un estudiante de nivel secundaria encuentra complejo relacionar la aplicación en la vida cotidiana, del álgebra y geometría (como ejemplo utilizando los ángulos). Soportado en el advenimiento del IoT y las TICs en particular a las aplicaciones móviles, en la Figura 7 se muestra un prototipo del control de la posición en grados de un servomotor, mediante una aplicación móvil (App) desarrollada en App inventor empleando el protocolo IEEE 802.15 (Bluetooth) para la comunicación.
Aplicaciones reales de este tipo de sistemas se encuentran relacionados con la domótica al controlar la posición de una puerta o de una antena, lo que hace discernir al estudiante de educación básica, la aplicación práctica del fundamento teórico a la vida cotidiana. Finalmente, con el mismo enfoque las mismas herramientas para el desarrollo de la App, comunicación y PCB, en la Figura 8 se presenta un prototipo para el control de un foco mediante una aplicación móvil.

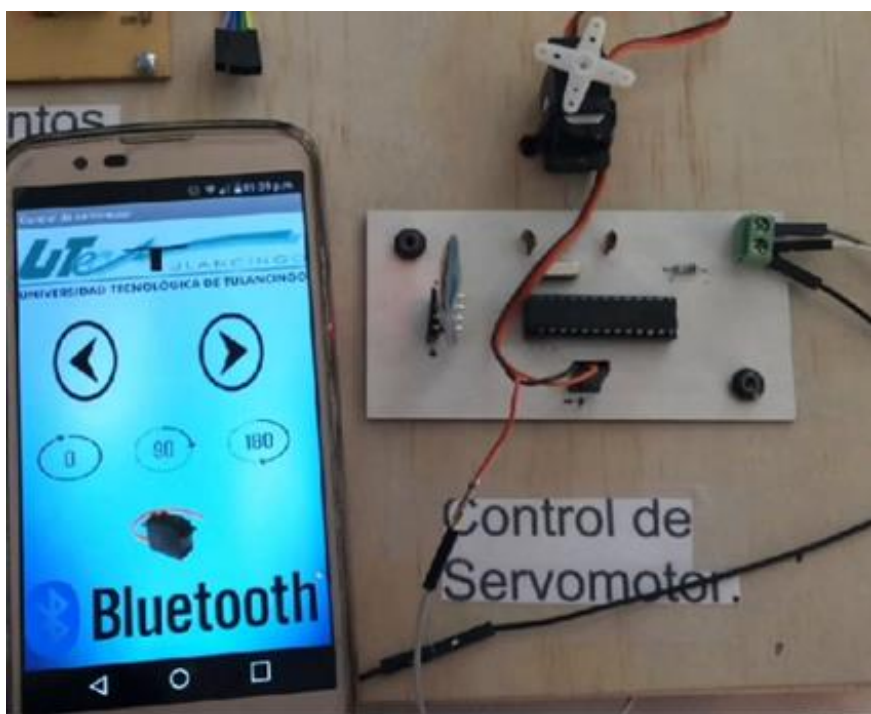

Figura 7: Sistema de control de la posición en grados de un servomotor mediante una aplicación móvil (Roldán-Aguirre, 2017).

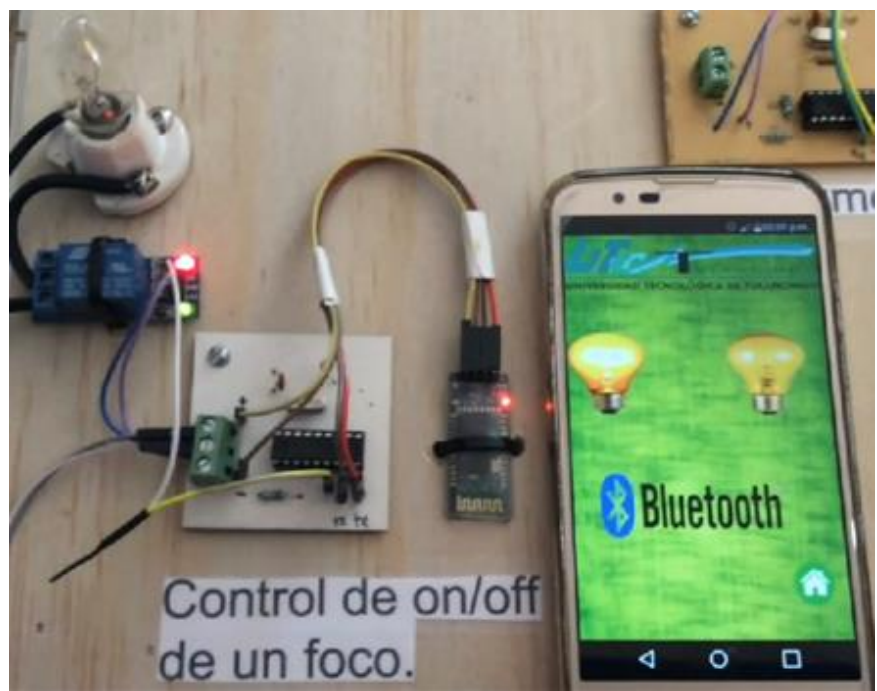

Figura 8: Sistema de control de foco mediante una aplicación móvil (Roldán-Aguirre, 2017).

La forma más común de visualizar esta aplicación en la vida real es en las casas y edificios inteligentes (domótica). No es lejana la posibilidad de que un estudiante de secundaria pueda usar estas herramientas para desarrollar sus propios sistemas. Es claro que el éxito de los profesionistas hoy en día es saber qué se hace con lo que se sabe.

Para complementar de manera pedagógica y didáctica el desarrollo de los prototipos con un enfoque de enseñanza mecatrónica, se realizó un manual de prácticas para cada uno de los sistemas que incluye: Nombre de la práctica, descripción del sistema, objetivo, materiales, fundamentación teórica y 
procedimiento interactivo. De manera general, los contenidos que se plantean abordar, basados en los aprendizajes clave de los programas de estudio de la SEP (2011) considerados en las guías para las materias de ciencias (2do grado) y matemáticas $(1,2$ y 3 grado):

- Matemáticas: Aritmética, trigonometría, geometría, estadística y álgebra.

- Ciencias: Física, metrología, variables físicas, electricidad y magnetismo, electrónica y mecánica.

- Tecnología: Informática, lógica, aplicaciones móviles, diseño y diagramas de flujo.

Los contenidos pueden ser evaluados directa e indirectamente mediante el desarrollo y utilización de los prototipos.

\section{Resultados y discusión}

En este apartado se describen de manera cualitativa los resultados más significativos de la implementación del enfoque en diversos escenarios, inicialmente mediante la puesta en marcha de la prueba piloto del enfoque de mecatrónica educativa para la educación básica, que se realizó en la escuela secundaria técnica ESTIC No. 0054, "Ignacio M. Altamirano", durante el ciclo escolar 2016-2017, ligado a la estadía profesional de un alumno del Programa Educativo de Ingeniería en Mecatrónica, miembro de este grupo de investigación. Durante la impartición del taller de robótica (30 hrs/semana total) se abordó el curso articulado a los contenidos de los temarios de ese nivel educativo a cuatro grupos de 30 alumnos aproximadamente, durante el ciclo escolar. Los grupos se constituyeron por 22 hombres y 13 mujeres de primer grado, 27 hombres y 15 mujeres de segundo y por 30 hombres y 17 mujeres de tercero.

En la Figura 9, se presenta evidencia fotográfica de dos alumnas usando el módulo de transmisor inalámbrico dentro del taller impartido.

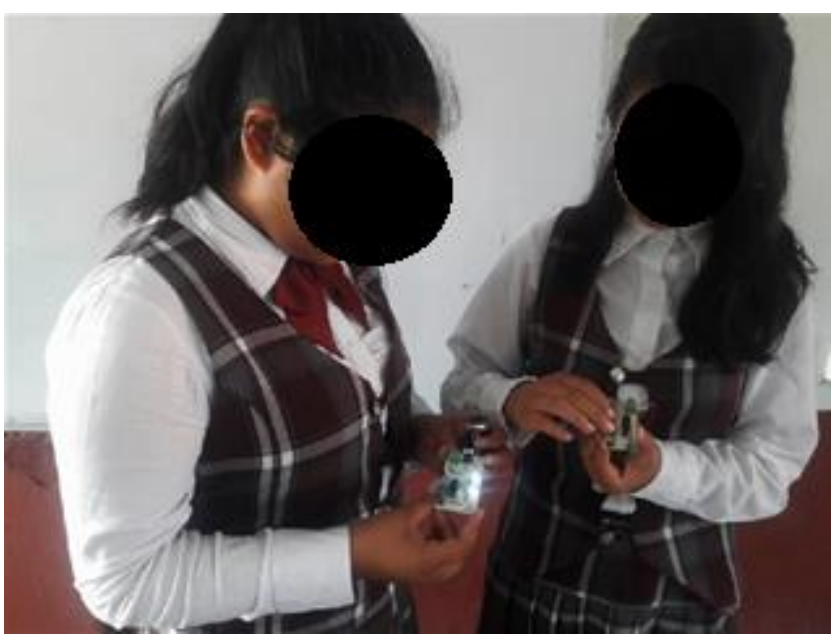

Figura 9: Alumnas de secundaria usando los módulos de transmisor inalámbrico (Elizalde-Huerta, 2017).

Cabe mencionar que los estudiantes fueron evaluados con la rúbrica diseñada para ello y se calificaron de acuerdo con su desempeño en el taller. Se puede aducir que la implementación del enfoque de mecatrónica educativa implantado en el taller de robótica, coadyuvan en el desarrollo de las competencias (habilidades, destrezas y valores) básicas en el currículo de la enseñanza secundaria como son: i) competencia en comunicación lingüística, al tener que interpretar y describir la teoría y principio de funcionamiento de los prototipos; ii) Competencia matemática, mediante el cálculo matemático empleado durante el taller; iii) Competencia en el conocimiento y la interacción con el mundo físico, la cual es el elemento central de este proyecto de aplicación; iv) Tratamiento de la información y competencia digital, inherente al ámbito de la tecnología propuesta en el modelo y v) Competencia social y ciudadana, mediante el fomento de valores individuales y trabajo en equipo.

Es de destacar, que el género de los estudiantes no es excluyente en el aprovechamiento y gusto por la tecnología y en particular por la mecatrónica. Hombres y mujeres mostraron el mismo desempeño e interés. Los modelos propuestos, son intuitivos para los usuarios finales y cualquier estudiante los puede manipular con el apoyo de una guía rápida del manual y de un profesor. La Figura 10 muestra evidencia fotográfica de la manipulación del brazo robótico y del módulo de detección de presencia ultrasónico.
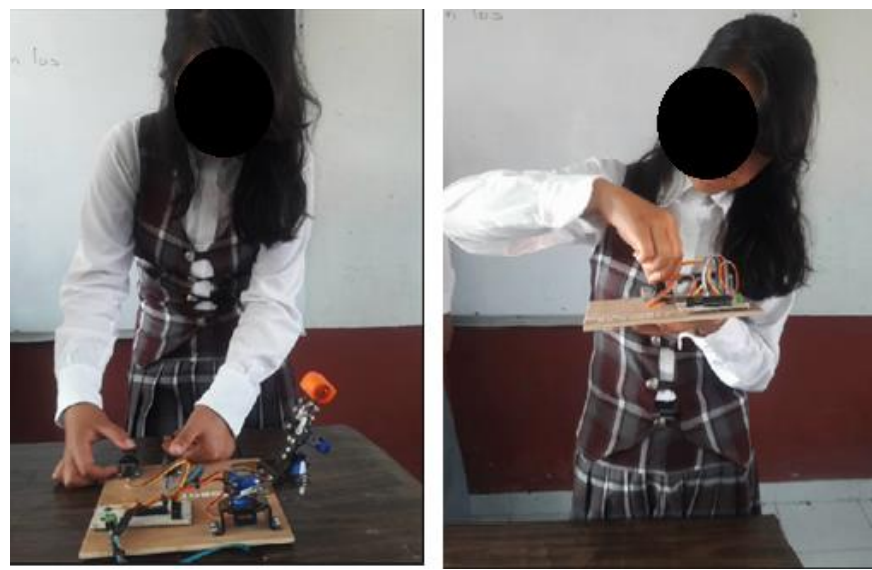

Figura 10: Uso de: izquierda, Brazo robótico manipulador y derecha, Módulo de detección de presencia ultrasónico (Elizalde-Huerta, 2017).

De igual manera, se puede aducir que la implantación de esta propuesta coadyuva al aprendizaje significativo mediante el enfoque constructivista basado en competencias. Además, de manera notable se observó que el desarrollo y uso de estos prototipos estimuló las distintas inteligencias: lógico matemática, espacial, kinestésica, intrapersonal e interpersonal, durante el proceso cognitivo desarrollado de los alumnos. Por ejemplo, un ángulo es la porción indefinida de plano limitada por dos líneas que parten de un mismo punto y cuya abertura puede medirse en grado, lo cual es difícil de conceptualizar. Sin embargo, en el prototipo de brazo robótico se ilustra didácticamente este concepto en el mundo real.

En relación con el grado de madurez tecnológica del modelo implementado en el taller, de acuerdo con la metodología TRL, se ha determinado que el enfoque de educación mecatrónica propuesto en este trabajo se ubica en el nivel 7: comisionamiento de sistemas. Dicho nivel se refiere al prototipo completo demostrado en ambiente relevante, ya que se ha demostrado que la tecnología funciona y opera a escala pre-comercial y se han identificado las cuestiones de la fabricación y operaciones finales.

Como se mencionó en la metodología después de haber obtenido los resultados significativos mediante la prueba piloto y la evaluación de madurez tecnológica del enfoque, se realizó la propuesta a la SEPH para escalarlo a más instituciones secundarias. En 2018 se iniciaron los trabajos con la impartición 
del curso: Fortalecimiento de la enseñanza de las matemáticas, ciencias naturales y tecnologías, a 33 profesores (18 hombre y 15 mujeres) de educación básica secundaria del estado de Hidalgo del que se muestra evidencia fotográfica en la Figura 11.

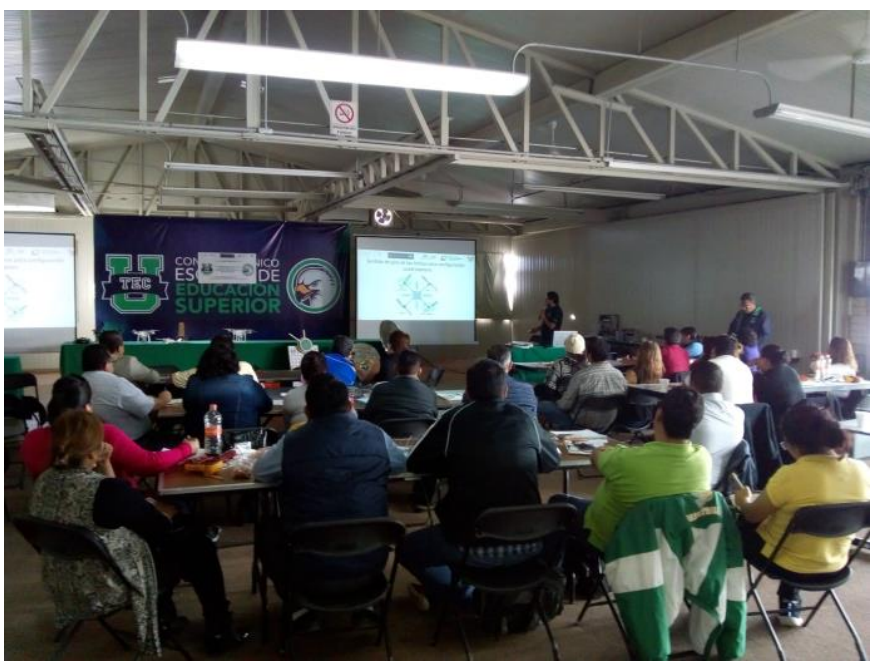

Figura 11: Evidencia fotográfica del curso Fortalecimiento de la enseñanza de las matemáticas, ciencia y tecnología impartido a profesores de nivel secundaria de la SEPH.

Inicialmente, se realizó una prueba diagnóstica para determinar el grado de conocimiento de los temas planteados en el taller, lo que permitió determinar la necesidad del curso con base en la evaluación (4/10) en promedio. Los resultados obtenidos al finalizar el curso se validaron mediante la aplicación de la misma prueba diagnóstica y existió una mejora considerable en la calificación (8/10) en promedio. En 2019, se dio continuidad al proyecto.

En la actualidad, el avance tecnológico y la utilidad de estos prototipos educativos han llevado a que existan empresas dedicadas al desarrollo de sistemas para la educación tecnológica, con el objetivo de que en las escuelas se capacite a los estudiantes para la inserción laboral en su ámbito. Tal es el caso de empresas muy exitosas como Festo ${ }^{\circledR}$, Lorenzo ${ }^{\circledR}$, ABB $®$, Siemens ${ }^{\circledR}$, National Instruments ${ }^{\circledR}$ entre otras, las cuales han provisto al mercado educativo de equipos muy buenos, pero generalmente muy costosos. Los resultados obtenidos en términos de costos de producción ( $\$ 500.00 \mathrm{MXN}$ en promedio por cada prototipo) y de implementación de los prototipos descritos, permiten determinar la factibilidad financiera y tecnológica de manera altamente competitiva, con respecto a los kits comerciales que ofrece Steren $\AA$, Electan $®$, Arduino $®$, Sostronic $₫$, Robótica Educativa de México, S. A. de C. V., entre otros. Estos, aunque son accesibles, son de propósito general y no establecen la articulación de manera directa con los contenidos del currículo de educación básica secundaria. Además, no promueven el conocimiento activo sino pasivo de los alumnos; en el sentido que sólo se debe usar y no se propone el aprender a aprender en la mayoría de los casos.

Debido a que los contenidos temáticos a nivel secundaria, en su mayoría, no contemplan la materia de programación, esto representa una limitante, en el sentido de que los estudiantes de dicho nivel carecen del conocimiento previo, requerido para programar y configurar los dispositivos y aplicaciones pertenecientes a los prototipos presentados en este trabajo, mediante el uso de lenguajes de bajo nivel, como $\mathrm{C}, \mathrm{C}++$, assembler, etc. Sin embargo, a través de herramientas que permiten programación a alto nivel de código abierto, como diagramas de flujo y de bloques, es posible que los estudiantes tengan un alto grado de interacción y comprensión de la lógica de programación usada en cada firmware y software, de los circuitos y aplicación usados en los kits didácticos, además, de permitir que los profesores transmitan con mayor rapidez y facilidad conceptos abstractos ejemplificados por medio de soluciones mecatrónicas.

\section{Conclusiones y trabajo futuro}

La propuesta didáctica para educación básica secundaria basada en educación mecatrónica de bajo costo presentada en este trabajo permite, a través de sus resultados preliminares, determinar la factibilidad tecnológica y metodológica para ser escalada a más escuelas del subsistema en el estado, incrementando las competencias de los estudiantes. La mecatrónica educativa coadyuva a la transición al paradigma de la educación 4.0 y cumple con las tendencias a nivel mundial en términos de la innovación educativa. Con el desarrollo de este enfoque, nuestra institución se manifiesta como una de las primeras en centrar sus esfuerzos en el desarrollo de este enfoque.

Este proyecto contribuye a las iniciativas de innovación del modelo educativo que la SEPH plantea, en donde los niños, niñas y jóvenes aprenden a aprender con una educación de calidad, un perfil claro en la articulación del currículo en todos los niveles educativos, centrado en los aprendizajes clave y que los maestros se formen continuamente de acuerdo a sus necesidades y las del entorno.

El desarrollo de esta investigación también advierte el elevado impacto educativo, mediante la articulación de contenidos, aprendizajes centrados, integración de todos los niveles educativos, mejoramiento elevado de las habilidades y conocimientos en las disciplinas científicas y tecnológicas del sistema de educación básica, pudiendo motivar el aprendizaje en el estudiante. Asimismo, como la promoción de la cultura del emprendimiento y la ciencia es muy importante que el capital humano formado en las instituciones de educación superior, asî como los científicos y tecnólogos atiendan problemáticas reales del entorno, mediante el desarrollo de proyectos como el planteado en este trabajo.

El trabajo futuro del enfoque didáctico basado en mecatrónica educativa consiste en implementar la prueba en otras instituciones de educación básica secundaria y hacer un análisis del aprovechamiento e impacto del mismo como una siguiente etapa; basado en un diseño experimental formal e instrumentos de validación, para determinar la satisfacción y beneficio de los estudiantes. Además, se plantea presentar una descripción detallada de la relación directa con materias de cada grado de nivel secundaria, así como sus respectivos tópicos del enfoque propuesto. Otro paso fundamental será la articulación de más niveles educativos; para el fortalecimiento y promoción de estas estrategias. Por ejemplo, los niveles de educación media superior y superior. Asimismo, esta propuesta se puede adaptar a otras áreas de la educación y disciplinas de formación.

\section{Agradecimientos}

En este trabajo, los estudiantes Elizalde-Huerta C. I. y RoldánAguirre J. que, mediante su Estadía de Ingeniería en Mecatrónica, participaron en el desarrollo e implementación de algunos de los prototipos mecatrónicos empleados en esta investigación y con el trabajo ejecutado se titularon.

\section{Referencias}

Cibrián-Anaya Zibrian Ricardo, Luque-Vega Luis Fernando y López-Neri Emmanuel. (2019). Transición de la robótica educativa: Caso de estudio: Ladrillo inteligente EV3 y Control Lógico Programable, Universo de la 
Tecnología, ISSN:2007-1450, Año 12, V1, Edición 34, sep-dic 2019, 19 22.

Dawes, J., Stocchi, L. \& Dall'Olmo-Riley, F. (2020). Over-time variation in individual's customer satisfaction scores. International Journal of Market Research, 62(3), 262-271.

Elizalde-Huerta, C. I. (2017). Kit de prácticas para fundamentos de robótica. [Tesis de Ingeniería en Mecatrónica, Universidad Tecnológica de Tulancingo]. Repositorio digital UTEC Tulancingo.

Gobierno del Estado de Hidalgo. (2016). Plan Estatal de Desarrollo, 20162022.

Halinen, I. \& Järvinen, R. (2008). Towards inclusive education: the case of Finland. Prospects, 38(1), 77-97.

Hussin, A. A. (2018). Education 4.0 made simple: Ideas for teaching. International Journal of Education and Literacy Studies, 6(3), 92-98.

Kananoja, T. A. P. A. N. I. (2009). Technology education in general education in Finland. International handbook of research and development in technology education, 51-64.

Kawamoto, Mari. (2015). Scientific and Technical Education in Japan's Junior and Senior High Schools National Institute for Research Advancement (NIRA).

King, John B. \& Russell Shilling. (2016). STEM 2026, A Vision for Innovation in STEM Education U.S. Department of Education, Office of Innovation and Improvement.

Krach, Keith. (2017). 13 de las últimas tendencias en tecnología educativa, Cofundador y CEO Ariba, presidente de Purdue University, presidente de Angie's y vicepresidente de General Motors, consultado por última vez 7 de abril de 2021, https://medium.com/@KeithKrach/13-of-thelatest-trends-in-educational-technology-e2368e $36 \mathrm{f7a}$ a
Lonka, Kirsti. (2020). Aprendizaje extraordinario Finlandia, Colección Educación Siglo del Hombre Editores.

Universidad de los Andes/Ediciones Uniandes. Colombia.

Lopez-Garcia, T. J., Alvarez-Cedillo, J. A., Sanchez, T. A., and VicarioSolorzano, C. M. (2019). Review of trends in the educational model of distance education in Mexico, towards an education 4.0. Computer Reviews Journal, 3, 111-121.

Luque-Vega, Luis F., López-Neri, Emmanuel, Santoyo, Anayeli, Ruíz-Duarte, Jorge \& Farrera-Vázquez, N. (2019). Educational methodology based on active learning for Mechatronics Engineering students: towards educational mechatronics. Computación y Sistemas, Vol. 23, No. 2, 2019, 325-333. doi: 10.13053/CyS-23-2-3196.

Olechowski, A. L., Eppinger, S. D., Joglekar, N., \& Tomaschek, K. (2020). Technology readiness levels: Shortcomings and improvement opportunities. Systems Engineering, 23(4), 395-408).

Roldan-Aguirre, J. (2017). Desarrollo de aplicación móvil para control de sistema mecatrónico. [Tesis de Ingeniería en Mecatrónica, Universidad Tecnológica de Tulancingo]. Repositorio digital UTEC Tulancingo.

Scolari, Carlos A. (2018). Adolescentes, medios de comunicación y culturas colaborativas. Fundación Colección Ceibal. ISBN 978-84-09-00293-1, 978-84-09-00292-4. Consultado 07042021. Disponible en https://digital.fundacionceibal/jspui/handle/123456789/247

Secretaria de Educación Pública. (2017). Resumen ejecutivo de la Reforma educativa Consultado por última vez el 24 de septiembre de 2017 en el sitio:

http://seduc.edomex.gob.mx/sites/seduc.edomex.gob.mx/files/files/Padr es\%20de\%20familia/Resumen_Ejecutivo_de_la_Reforma_Educativa.p df. 